\title{
红外热成像在乳腺疾病检测的应用研究
}

\author{
曾鉦妮，邓方阁 \\ (广州医科大学附属第一医院广州呼吸健康研究院 \\ 呼吸疾病国家重点实验室国家呼吸系统疾病临床医学研究中心，广东 广州 510120）
}

\begin{abstract}
摘要: 乳腺疾病已严重危害女性身心健康, 其中乳腺癌更位居全球范围内女性癌症发病率和死亡瘁首 位, 因此乳腺癌的早期发现意义重大。传统结构影像学早期检测疾病具有一定局限性, 而红外热成像 作为功能成像技术可为乳腺癌的早期篇查提供有效线索。因此本文主要就红外热成像在乳腺疾病的早 期检测及预后评估的应用价值进行综述。
\end{abstract}

关键词: 红外热成像; 乳腺疾病; 早期发现; 预后评估

中图分类号: R445.7; R737.9 文献标志码：A 文章编号：1001-8891(2020)05-0501-05

\section{Application of Infrared Thermal Imaging in Breast Disease Detection}

\author{
ZENG Jingni, DENG Fangge \\ (National Clinical Research Center for Respiratory Disease, State Key Laboratory of Respiratory Disease, Guangzhou Institute of \\ Respiratory Health, The First Affiliated Hospital of Guangzhou Medical University, Guangzhou 510120, China)
}

\begin{abstract}
Breast diseases are a threat to the physical and mental health of women. The occurrence of breast cancer and mortality resulting from it ranks the highest among cancer cases in women. Hence, early detection and diagnosis of breast cancer becomes extremely important. Traditional structural imaging has some limitations in early detection of diseases, while infrared thermal imaging, as a functional imaging technology, can provide effective clues for early screening of breast cancer. This work mainly reviews the usefulness of employing infrared thermal imaging in the early detection and prognosis evaluation of breast diseases.
\end{abstract}

Keywords: infrared thermal imaging, breast disease, earlydetection, prognosis evaluation

\section{0 引言}

乳腺疾病是危害女性健康的常见疾病类型之一, 随着生活方式、社会环境等因素的变化, 乳腺疾病的 发病人数逐渐增多。近年来, 据多项统计数据显示乳 腺癌发病率和死亡率逐年上升, 且发病年龄呈年轻化 趋势 ${ }^{[1]}$ 。国际癌症研究机构数据显示, 2018 年全球乳 腺癌发病率和死亡率仍居女性癌症首位 ${ }^{[2]}$ 。乳腺恶性 疾病的早期发现、早期诊断意义重大，更是降低其死 亡率及改善预后的关键。

乳腺疾病的影像学检查方法主要分为结构性影 像和功能影像两种, 前者主要包括乳腺 $\mathrm{X}$ 线钼靶 (mammography, MG)、核磁共振成像 (magnetic resonance imaging, MRI) 、超声（ultrasound, US）, 后者主要为红外热成像技术 (infrared thermal imaging, IRTI）。乳腺 X 线钿靶对致密型乳腺病灶 的检出敏感性较差, 并可带来一定的辐射暴露风险, 同时在检查过程中需压迫乳房会给患者带来不适感。 MRI 的特异性相对较低且价格较昂贵、检查耗时长, 不作为常规影像检查。超声是目前国内乳腺检查最常 用的影像方法, 在判断病灶的性质、位置、大小、数 目及血流情况等方面具有独有优势，但极依赖于设备 及操作医生的熟练度和诊断经验。红外热成像技术是 近几十年兴起的功能成像技术, 具有无创、无辐射、 快速、灵敏等优点, 已被应用于多种疾病的临床辅助 诊断, 本文主要就红外热成像在乳腺疾病的早期检测 及预后评估价值进行综述。

\section{IRTI 在乳腺成像的应用基础}


一般来说, 机体任何部位出现病变都是先出现局 部组织的代谢异常, 而对于恶性肿块或炎症病变, 由 于糖代谢增快、局部血流量增加、新生血管的形成以 及一氧化氮（nitric oxide，NO）和雌激素的生成，局 部热辐射也因此出现相应变化, 在人体体表表现为温 度的升高。在过去的几十年里, 越来越多的研究集中 于利用红外热像图来获得热生理学和皮肤温度之间 的相关性, 皮肤表面温度信息已被用于辅助诊断人体 的各种疾病, 包括外周血管疾病、乳腺癌、糖尿病神 经病变、发热篮查、疼痛、皮肤病、妇科疾病、针炎 治疗及法医学等 ${ }^{[3-4]}$ 。IRTI 是通过捕捉人体主动辐射 的红外能量进而转换成可视的温度图像来反映机体 组织器官血供及代谢情况, 基于病灶及其周围组织的 代谢活性与正常组织不同从而温度不同的原理来判 别疾病 ${ }^{[5-6]}$ 。现代红外热像仪的灵敏度已经可以检测到 $0.025^{\circ} \mathrm{C}$ 的温差, IRTI 作为安全可靠的功能成像方法 检测乳腺疾病, 其适用人群广, 包括所有年龄段女性、 孕妇、哺乳期妇女、假体植入者及术后患者, 也可用 于监测疾病治疗及了解腋窝情况 ${ }^{[7]}$ 。

\section{IRTI 检测乳腺疾病的临床应用价值}

乳腺疾病严重危害女性身心健康, 包括乳腺纤维 瘤、乳腺增生、乳腺炎、乳腺囊肿、乳腺癌等。乳腺 肿块按性质可分为囊性肿块及实性肿块, 因内部成分 不同二者的代谢率不同, 囊性病变缺乏血液供应不会 额外产生热量因此可区分于实性病变。Zare I. 和 Zadeh 等人 ${ }^{[8-9]}$ 通过对 60 名疑有乳腺疾病患者的热像图结果 与超声、活检结果对比研究, 发现 IRTI 识别实性肿 块和囊性肿块的准确性分别为 $91.89 \% 、 92.30 \%$, 认 为热像图可区分实性低回声肿块和囊性肿块。

乳腺实性肿块中以乳腺癌危害最为严重, 癌症细 胞的生长不受控制, 当癌前细胞及癌细胞快速生长 时, 为了满足对营养物质及氧气的需求, 癌症组织会 产生新生血管、局部代谢加快导致相应部位组织温度 升高。1956 年Lawson 首次将 IRTI 用于乳腺癌的检测, 发现病变组织处的皮肤温度高于正常乳腺组织 ${ }^{[10]}$ 。 1963 年 Lawson 和 Chughtai 分析这是由于癌性组织代 谢活动增加产热增多及新生血管生成增加的缘故 ${ }^{[11]}$ 。 基于此原理, 有学者就 IRTI 对乳腺癌发生的预警作 用进行了研究。Gautherie 等 ${ }^{[12]}$ 的研究发现约 $35 \%$ 热 像图异常的健康女性在 5 年内罹患乳腺癌, 认为异常 热像图在预测乳腺癌发生中的作用比一级家族史更 重要, 异常的热像图是未来患乳腺癌风险的最重要的 标志。Amalric 等人 ${ }^{[13]}$ 在长达 10 年的时间里, 使用热 像仪对 61000 名女性进行了篮查, 结果发现对于 $60 \%$
的病例而言热像图最早提供乳腺癌的信息。也有报道 称热像图能够比乳腺 $X$ 线钼靶提早 $8 \sim 10$ 年预警乳腺 癌的发生 ${ }^{[14]}$ 。以上研究表明, IRTI 在预测乳腺癌发生 中有一定价值。由于 IRTI 对乳腺血管成像的独特能 力, 长期随访可得到非常早期的预警信息, 热像图是 目前已知最早预测乳腺癌进展的指标。

乳腺癌最常见的热像图特征为乳房温度分布明 显不对称, 可见局灶性热区, 血管形态不规则增粗迁 曲, 温度梯度可区分良恶性肿瘤, 目前多数认为较高 的温度梯度 $\left(>1{ }^{\circ} \mathrm{C}\right)$ 可作为恶性肿瘤的判断指标, 较低的温度与乳腺良性疾病有关。图 1 和图 2 为经活 检证实的乳腺癌患者的红外热像图 ${ }^{[15-16]}$ 。迄今, 越来 越多的研究证明 IRTI 可用于乳腺恶性疾病的早期篎 查、良恶性判断及辅助诊断。Rassiwala 等 ${ }^{[17]}$ 对 1008 例 20 60 岁未诊断为乳腺癌的女性进行 IRTI 检查, 发现 IRTI 对局部温差超过 $3^{\circ} \mathrm{C}$ 的乳腺癌具有较高的敏

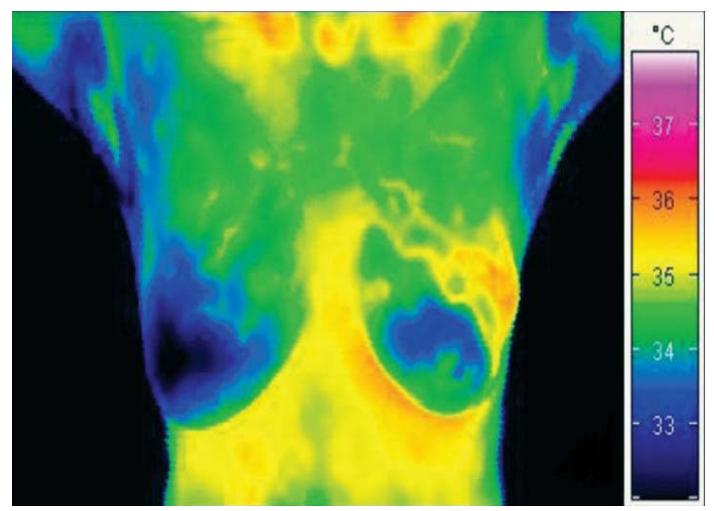

图 158 岁女性右乳腺癌 (乳晕下区) 热像图

Fig.1 Thermogram of a 58 years old female having right breast cancer (sub-areolar region)

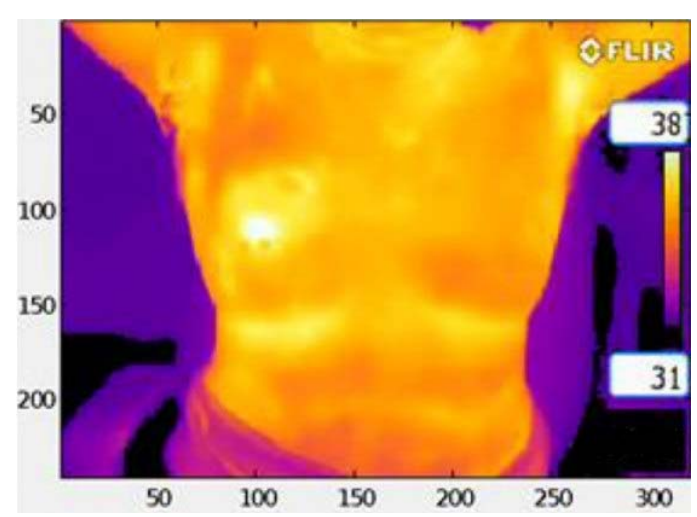

图 254 岁左乳肿块女性, 热像图显示左乳外上象限温度高于 右乳, 活检证实为恶性肿瘤

Fig.2 A 54-year-old woman with left breast mass showed that the temperature of the upper outer quadrant of the left breast was higher than that of the right breast and was confirmed by biopsy as a malignant tumor 
感度和特异度（分别达 $97.6 \% 、 99.17 \%$ ），阳性预测 值和阴性预测值分别为 $83.67 \% 、 99.89 \%$, 认为 IRTI 可作为乳腺癌篮查的有用工具。Sarigoz 等 ${ }^{[18]}$ 将 54 例 触诊有乳腺肿块的患者的 IRTI 结果与超声、活检结 果进行对比, 认为 IRTI 可用于鉴别乳腺肿块的良恶 性，其灵敏度和特异度分别为 $95.24 \% 、 72.73 \%$, 在 乳腺浸润性导管癌、纤维腺瘤及囊肿的鉴别中亦具有 一定作用。随着 IRTI 在乳腺的应用, 近年来学者们 致力于对图像处理进行技术突破以提高其乳腺癌篮 查的能力, Morales-Cervantes 等人 ${ }^{[19]}$ 利用计算机辅助 诊断来研究 IRTI 检测乳腺癌的有效性, 提出了利用 自动化程序对热像图进行图像分类评分的方法, 该研 究共纳入 206 例疑似乳腺癌患者, 通过分析病灶处与 对侧镜像处乳房的温度差及乳房的血管密度, 对比病 理结果, 发现 IRTI 的灵敏度可达 $100 \%$, 特异度为 $68.68 \%$, 阳性预测值为 $11.42 \%$, 阴性预测值为 $100 \%$, 再次证实 IRTI 是一种较好的乳腺癌笁查工具。Ekici 等 ${ }^{[20]}$ 利用贝叶斯算法优化的卷积神经网络 (Convolutional Neural Networks, CNNs) 对 140 例乳 腺癌女性进行数据分析, 将乳腺热像图分为正常或可 疑两类, 其篮查准确率达到 $98.95 \%$ 。以上研究均表明 IRTI 早期篎查乳腺癌的灵敏度较高, 可作为乳腺癌辅 助筛查工具用于临床。

同时不少学者将 IRTI 与超声、乳腺 X 线钿靶对 乳腺疾病的诊断有效性进行对比研究来明确其临床 实际应用效能。Yao 等 ${ }^{[21]}$ 对 2036 例乳腺疾病患者行 乳腺 $\mathrm{X}$ 线钿靶、超声及 IRTI 检查, 以术后病理结果 为标准, 发现对于 $2 \mathrm{~cm}$ 以下的病灶 IRTI 的准确性高 于超声和钼靶（分别为 $97.1 \% 、 95.8 \% 、 96.1 \%$ ），而 $>2 \mathrm{~cm}$ 的病灶 IRTI 与超声准确性相当, 高于 X 线钼 靶的准确性, 因此认为 IRTI 在乳腺癌大规模人群的 早期篮查中具有较高应用价值。Alikhassi 等 $^{[5]}$ 对 78 例女性患者进行乳腺 IRTI 与超声对比研究, 发现 IRTI 的灵敏度、特异度和准确率依次为 $85.7 \%$ 、78.5\%、 $78.8 \%$, 超声的灵敏度、特异度和准确率依次为 $100 \%$ 、 $95.3 \% 、 95.5 \%$, 在诊断效能上 IRTI 仍低于超声, 认
为 IRTI 仅可作为诊断乳腺恶性病变的辅助手段。 Omranipour 等 ${ }^{[22]}$ 则对 132 名拟行乳腺穿刺活检的患者 进行钼靶及 IRTI 检查, 发现二者的灵敏度相差不大 （钿靶、IRTI 分别为 $80.5 \% 、 81.6 \%$ ），但钿靶的特异 度、阳性预测值、阴性预测值及准确性较 IRTI 稍高 （钿靶依次为 $73.3 \% 、 85.4 \% 、 66 \% 、 76.9 \%$, IRTI 依 次为 $57.8 \% 、 78.9 \% 、 61.9 \% 、 69.7 \%$ ），认为 IRTI 虽 可用于乳腺癌的早期辅助诊断但不能代替乳腺 $\mathrm{X}$ 线 钿靶。而在 Kolaric 等 ${ }^{[23]}$ 的研究中 IRTI 的灵敏度已到 达 $100 \%$, 高于 X 线钿靶的灵敏度 $(85 \%)$ 。以上研 究虽对 IRTI 与超声、钼靶的诊断有效性报道不一, 但均表明 IRTI 可用于乳腺恶性病变的辅助诊断, 但 仍不能作为其他检查方法的替代手段，几种方法联合 应用更是有望提高诊断准确率。

目前红外热成像技术作为乳腺癌篮查或辅助诊 断工具得到了广泛认可（表 1)，但仍有部分学者存 在不同意见, 这主要是由于不同作者研究所得到的 IRTI 特异性和灵敏度不一致所致。Vreugdenburg 等 人 $^{[24]}$ 对 7 个生物医学数据库进行检索, 观察到 IRTI 灵敏度 $(0.25 \sim 0.97)$ 和特异度 $(0.12 \sim 0.85)$ 的异质 性较大, 因此认为没有足够证据支持 IRTI 作为篮查 手段。Sajadi 等人 ${ }^{[25]}$ 也得出相似结论, 并认为与其他 诊断技术相比，热成像诊断价值不高。关于这些报道 中结果异质性归因于各项研究中使用的设备性能不 同, 以及用于将红外热图分类为正常或疾病的算法的 差异, 同时作者也指出纳入的部分文献的研究质量或 研究方法不理想, 另外可能遗漏部分研究, 因此需谨 慎对待其结果。

\section{IRTI 在乳腺癌预后评估中的价值}

随着分子生物学研究的不断进展, 乳腺癌发生、 发展及预后与各种分子生物学指标的关系已得到证 实, 乳腺癌相关预后因子在指导个体化诊断、治疗方 案选择、疗效检测及预后判断方面有重要意义。其中, 最常用的预后因子是雌激素受体 (estrogen receptor, ER）、孕激素受体 (progesterone receptor, PR) 和人

表 1 IRTI 检测乳腺癌效能结果 Table 1 IRTI Results of Breast Cancer Detection

\begin{tabular}{llllll}
\hline \multirow{2}{*}{ References } & \multirow{2}{*}{ Year } & \multirow{2}{*}{ Samples } & \multicolumn{3}{c}{ IRTI } \\
\cline { 4 - 6 } & & & Sensitivity & Specificity & Accuracy \\
\hline 23 & 2013 & 26 & $100 \%$ & $79 \%$ & $92 \%$ \\
17 & 2014 & 1008 & $97.6 \%$ & $99.17 \%$ & - \\
21 & 2014 & 2036 & $84.4 \%$ & $94.0 \%$ & $91.7 \%$ \\
22 & 2016 & 132 & $81.6 \%$ & $73.3 \%$ & $76.9 \%$ \\
18 & 2018 & 54 & $95.24 \%$ & $72.73 \%$ & - \\
19 & 2018 & 206 & $100 \%$ & $66.86 \%$ & - \\
5 & 2018 & 78 & $85.7 \%$ & $78.5 \%$ & $78.8 \%$ \\
\hline
\end{tabular}


类表皮生长因子受体-2（human epidermal growth factor receptor-2, HER-2)。ER 阴性、PR 阴性和 HER-2 阳性的肿瘤侵袭性较强, 生长速度快, 需要更多新生 血管生成供应其生长，从而导致局部温度升高。同时 雌激素及孕激素可扩张周围血管, 并可通过增加 $\mathrm{NO}$ 的生成调节血管舒张, 引起整个乳房温度的改变。国 外学者已经就乳腺癌的红外热成像与组织病理学、免 疫组化因子的相关性展开了研究以明确 IRTI 在预测 乳腺癌预后的价值。Wang J. 等 ${ }^{[26]}$ 通过对 163 名乳腺 癌患者共 171 例病灶的红外热像图特征与激素受体表 达情况关系的研究发现, 乳腺癌病灶区与镜像部位的 温差与 $\mathrm{ER} 、 \mathrm{PR}$ 表达呈负相关, 同侧乳房病灶部位与 相邻正常乳腺组织温差与 PR 表达呈负相关, 而 HER-2 与热像图特征无相关性。Zore 等人 ${ }^{[27-29]}$ 的几项 研究表明 ER-、PR-、HER-2+肿瘤病灶部位的温度较 ER+、PR+、HER-2-肿瘤高, ER 阴性对乳房整体温度 升高的影响较小, ER+患者患侧乳房的温度高于 ER患侧乳房。Mance 等人 ${ }^{[30]}$ 的研究也发现 $E R+$ 和 $P R+$ 肿瘤的温度较高, 而 HER-2 与乳腺温度无相关性。以 上研究表明激素受体表达情况与热像图之间有一定 相关性, 可通过热像图表现初步评估患者预后。然而, 另外有研究却认为激素受体表达情况与红外热像图 之间并无关联, 但与生长速度相关的预后指标与热图 密切相关 ${ }^{[31-32]}$ 。Ohsumi 等 ${ }^{[33]}$ 利用 IRTI 对 340 例无远 处转移的单侧浸润性乳腺癌患者进行预后评估, 分析 肿瘤部位与其对侧镜像部位的温差 $\Delta T$ 与各因素的关 系, 发现 $\Delta T$ 虽与雌激素受体表达情况无关, 但却与 肿瘤大小、组织学状态、核分级显著正相关, 对于淋 巴结阳性的乳腺癌患者, $\Delta T$ 可作为判断预后的有用 指标。以上多项研究表明乳腺癌激素受体表达情况与 热像图的关系尚无一致结论, 但由于目前该方面相关 研究文献较少, 因此红外热成像技术是否可用于评估 乳腺癌预后尚需更多的深入研究以明确其临床应用 价值。

\section{4 乳腺肿瘤特点对 IRTI 成像的影响}

体表温度易受环境和个体因素影响, 如血管及生 物活性的变化、组织传导的改变、内分泌因素。目前 研究认为在对检测环境和受检人员进行质控后, 乳腺 肿瘤的红外热像图主要受肿瘤自身特性影响, 如肿瘤 的大小、深度、血流丰富程度和组织学特征等。Yan Zhou 和 Herman ${ }^{[34]}$ 利用 COMSOL 软件评估了二维乳 腺模型中不同肿瘤位置和大小的表面热对比度, 发现 肿瘤的大小和深度是影响热成像最大热对比度的最 主要因素。Mance 等 ${ }^{[30]}$ 对 50 名经活检确诊为乳腺导
管癌的女性分析发现, 病变乳腺温度与肿瘤体积呈正 相关, 与肿瘤深度呈负相关。Gonzalez 等 ${ }^{[35]}$ 通过建立 乳腺及癌性肿瘤简化模型表明 IRTI 可检测到距离皮 肤 $7 \mathrm{~cm}$ 以上的 $3 \mathrm{~cm}$ 的肿瘤, 而对于小于 $0.5 \mathrm{~cm}$ 的肿 瘤仅距离皮肤表面较近时才能被检测到。Jiang 等人 ${ }^{[36]}$ 的研究则发现皮肤温度的改变通常只能在深度小于 $20 \mathrm{~mm}$ 的浅表肿瘤中发现。肿瘤深度小于 $20 \mathrm{~mm}$ 时可 使健乳与患乳温差增加 $1.5^{\circ} \mathrm{C}$, 深度在 $20 \mathrm{~mm}$ 以上时 其引起的表面温度差异不明显, 相对于肿瘤直径, 深 度对温度的影响更大。也有研究证实 IRTI 对直径 $2 \mathrm{~cm}$ 以下的肿瘤灵敏度高于其他影像学方法 ${ }^{[21]}$ 。Kandlikar 等 ${ }^{\left[{ }^{[7]}\right.}$ 也得出相似结论认为肿瘤深度是影响表面温度 的主要因素。而皮肤温度受皮肤血流量的影响, 肿瘤 周围组织温度也与肿瘤微血管密度正相关 ${ }^{[38]}$ 。这些研 究说明乳腺病灶表面温度主要与肿瘤位置和大小有 关, 相对于肿瘤直径, 肿瘤深度对乳腺表面温度的影 响更大, 对于一定深度的肿瘤, 红外热像图对其显示 不佳, 随着肿瘤靠近体表, 皮肤表面的热对比度增大。

\section{5 总结及展望}

作为功能成像技术, 红外热成像已成为乳腺疾病 检查的新方法, 可较早发现异常病变, 在一定程度上 弥补了结构影像学的不足。尽管红外热成像作为乳腺 疾病早期篮查的工具具有较高临床应用价值, 但仍不 能代替现有的乳腺疾病检查技术。与此同时，因其具 有操作简单、价格便宜、成像直观易于解读、精确灵 敏等优点, 适用于大规模的人群乳腺癌笁查, 而我国 人口基数大、人均收入普遍较低, 许多基层医院诊断 手段不足导致乳腺癌患者不能早期得到有效治疗而 延误病情，红外热成像的应用可以提高基层医院的医 疗诊断水平，帮助乳腺癌的早期发现，尤其对经费不 足但同时需要提高医疗水平的中、小型医院更是值得 推广。

\section{参考文献:}

[1] 师金, 梁迪, 李道娟, 等. 全球女性乳腺癌流行情况研究[J]. 中国肿 瘤, 2017, 26(9): 683-690.

SHI Jin, LIANG Di, LI Daojuan, et al. Epidemiological Status of Global Female Breast Cancer[J]. China Cancer, 2017, 26(9): 683-690.

[2] Bray F, Ferlay J, Soerjomataram I, et al. Global cancer statistics 2018 : GLOBOCAN estimates of incidence and mortality worldwide for 36 cancers in 185 countries[J]. CA Cancer J Clin, 2018, 68(6): 394-424.

[3] Lahiri B B, Bagavathiappan S, Jayakumar T, et al. Medical applications of infrared thermography: a review[J]. Infrared Physics \& Technology, 2012, 55(4): 221-235.

[4] Figueiredo A, Do N J, Malheiros F C, et al. Breast tumor localization using skin surface temperatures from a $2 \mathrm{D}$ anatomic model without 
knowledge of the thermophysical properties[J]. Computer Methods and Programs in Biomedicine, 2019, 172: 65-77.

[5] Alikhassi A, Hamidpour S F, Firouzmand M, et al. Prospective comparative study assessing role of ultrasound versus thermography in breast cancer detection[J]. Breast Disease, 2018, 37(4): 191-196.

[6] De Jesus G R, Oliveira L L V M, Das N L, et al. Accuracy and Reliability of Infrared Thermography in Assessment of the Breasts of Women Affected by Cancer[J]. J Med Syst, 2017, 41(5): 87.

[7] Milosevic M, Jankovic D, Milenkovic A, et al. Early diagnosis and detection of breast cancer[J]. Technology and Health Care, 2018, 26(4): 729-759.

[8] Zare I, Ghafarpour A, Zadeh H G, et al. Evaluating the thermal imaging system in detecting certain types of breast tissue masses[J]. Biomedical Research-India, 2016, 27(3): 670-675.

[9] Zadeh H G, Haddadnia J, Ahmadinejad N, et al. Assessing the potential of thermal imaging in recognition of breast cancer[J]. Asian Pac $J$ Cancer Prev, 2015, 16(18): 8619-8623.

[10] LAWSON R. Implications of surface temperatures in the diagnosis of breast cancer[J]. Can Med Assoc J, 1956, 75(4): 309-311.

[11] Lawson R N, Chughtai M S. Breast Cancer and Body Temperature[J]. Can Med Assoc J, 1963, 88(2): 68-70.

[12] Gautherie M. Thermobiological assessment of benign and malignant breast diseases[J]. American Journal of Obstetrics and Gynecology, 1983, 147(8): 861-869.

[13] Amalric R, Giraud D, Altschuler C, et al. Does infrared thermography truely have a role in present day breast cancer management?[J]. Biomed Thermology, 1982, 107: 269-278.

[14] Ng E Y, Ung L N, Ng F C, et al. Statistical analysis of healthy and malignant breast thermography[J]. J Med Eng Technology, 2001, 25(6): 253-263.

[15] Prasad S S, Ramachandra L, Kumar V, et al. Evaluation of efficacy of thermographic breast imaging in breast cancer: A pilot study[J]. Breast Disease, 2016, 36(4): 143-147.

[16] Umadevi V, Raghavan S V, Jaipurkar S. Framework for estimating tumour parameters using thermal imaging[J]. The Indian Journal of Medical Research, 2011, 134(5): 725.

[17] Rassiwala M, Mathur P, Mathur R, et al. Evaluation of digital infra-red thermal imaging as an adjunctive screening method for breast carcinoma: A pilot study[J]. International Journal of Surgery, 2014, 12(12): 1439-1443.

[18] Sarigoz T, Ertan T, Topuz O, et al. Role of digital infrared thermal imaging in the diagnosis of breast mass: A pilot study Diagnosis of breast mass by thermography[J]. Infrared Physics \& Technology, 2018, 91: 214-219.

[19] Morales-Cervantes A, Kolosovas-Machuca E S, Guevara E, et al. An automated method for the evaluation of breast cancer using infrared thermography[J]. EXCLI journal, 2018, 17: 989-998.

[20] Ekici S, Jawzal H. Breast Cancer Diagnosis Using Thermography and Convolutional Neural Networks[J]. Medical Hypotheses, 2020, 137: 109542.

[21] YAO X, WEI W, LI J, et al. A comparison of mammography, ultrasonography, and far-infrared thermography with pathological results in screening and early diagnosis of breast cancer[J]. Asian Biomedicine, 2014, 8(1): 11-19.

[22] Omranipour R, Kazemian A, Alipour S, et al. Comparison of the Accuracy of Thermography and Mammography in the Detection of Breast Cancer[J]. BREAST CARE, 2016, 11(4): 260-264.
[23] Kolarić D, Herceg Ž, Nola I A, et al. Thermography-a feasible method for screening breast cancer?[J]. Collegium Antropologicum, 2013, 37(2): 583-588.

[24] Vreugdenburg T D, Willis C D, Mundy L, et al. A systematic review of elastography, electrical impedance scanning, and digital infrared thermography for breast cancer screening and diagnosis[J]. Breast Cancer Research and Treatment, 2013, 137(3): 665-676.

[25] Sajjadi H S, Seyedin H, Aryankhesal A, et al. A systematic review on the effectiveness of thermography in diagnosis of diseases[J]. International Journal of Imaging Systems and Technology, 2013, 23(2): 188-193.

[26] WANG J, Shih T T, Yen R, et al. The Association of Infrared Imaging Findings of the Breast with Hormone Receptor and Human Epidermal Growth Factor Receptor 2 Status of Breast Cancer[J]. Academic Radiology, 2011, 18(2): 212-219.

[27] Zore Z, Boras I, Stanec M, et al. Influence of hormonal status on thermography findings in breast cancer[J]. Acta Clin Croat, 2013, 52(1): $35-42$.

[28] Zore Z, Filipović-Zore I, Stanec M, et al. Association of clinical, histopathological and immunohistochemical prognostic factors of invasive breast tumors and thermographic findings[J]. Infrared Physics and Technology, 2015, 68: 201-205.

[29] Zvonimir Z, Ivanka B, Irina F, et al. The impact of human epidermal growth factor receptor-2 status of invasive breast tumors on thermography findings[J]. Saudi Med J, 2012, 33(10): 1118-1121.

[30] Mance M, Bulic K, Antabak A, et al. The influence of size, depth and histologic characteristics of invasive ductal breast carcinoma on thermographic properties of the breast[J]. EXCLI Journal, 2019, 18: 549.

[31] Sterns E E, Zee B, SenGupta S, et al. Thermography. Its relation to pathologic characteristics, vascularity, proliferation rate, and survival of patients with invasive ductal carcinoma of the breast.[J]. Cancer, 1996, 77(7): 1324-1328.

[32] Head J F, Wang F, Elliott R L. Breast thermography is a noninvasive prognostic procedure that predicts tumor growth rate in breast cancer patients[J]. Annals of the New York Academy of Sciences, 1993, 698: 153-158.

[33] Ohsumi S, Takashima S, Aogi K, et al. Prognostic Value of Thermographical Findings in Patients with Primary Breast Cancer[J]. Breast Cancer Research and Treatment, 2002, 74(3): 213-220.

[34] ZHOU Y, Herman C. Optimization of skin cooling by computational modeling for early thermographic detection of breast cancer[J]. International Journal of Heat and Mass Transfer, 2018, 126: 864-876.

[35] Gonzalez F J. Infrared imager requirements for breast cancer detection[C]//Annual International Conference of the IEEE Engineering in Medicine and Biology Society, 2007: 3312-3314.

[36] JIANG L, ZHAN W, Loew M. Combined thermal and elastic modeling of the normal and tumorous breast[C]//Proc. SPIE, 2008: 6916.

[37] Kandlikar S G, Perez-Raya I, Raghupathi P A, et al. Infrared imaging technology for breast cancer detection - Current status, protocols and new directions[J]. International Journal of Heat and Mass Transfer, 2017, 108: 2303-2320.

[38] Yahara T, Koga T, Yoshida S, et al. Relationship between microvessel density and thermographic hot areas in breast cancer[J]. Surgery Today, 2003, 33(4): 243-248. 